\title{
Endovascular Treatment of Ruptured Vertebral Artery Dissecting Aneurysm in Fibromuscular Dysplasia
}

\section{Tratamento endovascular de aneurisma dissecante roto de artéria vertebral na displasia fibromuscular}

\author{
Luana Antunes Maranha Gatto ${ }^{10}$ Diego do Monte Rodrigues Seabra ${ }^{1}$ Jennyfer Paulla Galdino Chaves ${ }^{1}$ \\ Gelson Luis Koppe ${ }^{1}$ Zeferino Demartini J $r^{1}$ \\ ${ }^{1}$ Department of Neurosurgery and Interventional Neuroradiology, \\ Hospital Universitário Cajuru, Curitiba, PA, Brazil \\ Arq Bras Neurocir 2019;38:149-152.

\begin{abstract}
Address for correspondence Luana Antunes Maranha Gatto, MD, Departamento de Neurocirurgia e de Neurorradiologia Intervencionista, Hospital Universitário Cajuru, Rua São José, 300, Curitiba, PA, 80050350,
\end{abstract} \\ Brazil (e-mail: luanamaranha@yahoo.com.br).
}

\author{
Abstract \\ Keywords \\ - fibromuscular \\ dysplasia \\ - dissecting aneurysm \\ - endovascular \\ procedure \\ - carotid stenosis \\ - angioplasty \\ - postoperative \\ complications

\section{Resumo \\ Palavras-chave \\ - displasia fibromuscular \\ - aneurisma dissecante \\ - procedimento endovascular \\ - estenose carotídea \\ - angioplastia \\ - complicações pós operatórias}

Background Fibromuscular dysplasia (FMD) affects predominantly the cervical and renal arteries and may cause the classical angiographic pattern of string-of-beads. The diagnosis is increasing with the advances of imaging techniques.

Case Report A 37-year-old man presenting with subarachnoid hemorrhage due to a dissecting aneurysm of the vertebral artery was treated by angioplasty with stent, with good outcome. All of the cervical and renal arteries were diseased and showed dysplasia and/or ectasias.

Conclusions There are no guidelines or protocols to treat patients with FMD.

Introdução A displasia fibromuscular (DFM) afeta predominantemente as artérias cervicais e renais e pode causar o padrão angiográfico clássico de cordão de contas. O diagnóstico tem aumentado com os avanços das técnicas de imagem.

Relato de Caso Homem de 37 anos, apresentando hemorragia subaracnoidea por aneurisma dissecante da artéria vertebral, foi tratado por angioplastia com stent, com bom resultado. Todas as artérias cervicais e renais estavam doentes e apresentavam displasia e / ou ectasias.

Conclusões Não existem diretrizes ou protocolos para o tratamento de pacientes com DFM.

(1)Luana Antunes Maranha Gatto's ORCID is https://orcid.org/00000002-1940-2689.

received

January 21, 2019

accepted

March 20, 2019
DOI https://doi.org/

10.1055/s-0039-1688459. ISSN 0103-5355.
Copyright (e 2019 by Thieme Revinter

Publicações Ltda, Rio de Janeiro, Brazil
License terms

(c) $(1) \$$ 


\section{Introduction}

Fibromuscular dysplasia (FMD) is a group of idiopathic, nonatherosclerotic and noninflammatory diseases of the arterial walls leading to small- and medium-sized artery stenosis. ${ }^{1,2}$ Fibromuscular dysplasia can occur in any artery, although it is more prevalent in the renal and cervical arteries. ${ }^{3}$ We report a case of FMD presenting with subarachnoid hemorrhage (SAH) due to left vertebral artery (IfVA) dissection, treated successfully by angioplasty.

\section{Case Report}

A 37-year-old man presented with sudden headache and vomiting and was admitted 7 days after in the emergency room. He was admitted with mild stiff neck, Glasgow Come Scale 15, Hunt-Hess I, World Federation of Neurological Societies (WFNS) I. There was a medical history of high blood pressure (HBP) for 12 years controlled with amlodipine, and he denied head trauma, infection of the central nervous system (CNS), addictions, or a family history of cerebrovascular event. A computed tomography (CT) scan and a magnetic resonance imaging (MRI) exam of the head showed a SAH in the skull base cisterns and in the $4^{\text {th }}$ ventricle, Fisher IV (-Fig. 1). A digital subtraction angiography (DSA) evidenced two dissecting aneurysms in the V3 segment of the IfVA, one in the right petrous carotid portion, and a "string-of-beads" pattern with stenosis followed by dilatations in both internal carotid arteries (ICAs) (multifocal type), and in both V2 segments, as well as right

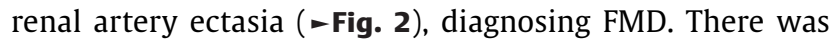
clinical worsening requiring mechanical ventilation due to urinary focus sepsis, non-biliary pancreatitis, and acute renal failure (both contrast-related), bilateral hydronephrosis grade II, and transient thrombocytopenia. After clinical improvement without the need for dialysis, on the 37th day postictus, a transluminal percutaneous angioplasty (TPA) of the dissecting aneurysm was performed under general anesthesia and full intravenous heparinization. The uneventful procedure was performed through the right femoral artery (rtFA), and two telescoped coronary stents

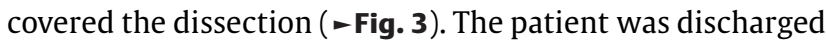
with no neurological deficit, using acetylsalicylic acid (ASA) and clopidogrel. A control DSA 8 months postsurgery showed exclusion of one IfVA aneurysm and decrease of the other one. The patient remained asymptomatic in a 1year follow-up.

\section{Discussion}

Three main types of renal FMDs were identified according to the arterial wall layer that is mainly affected: intimal $(\sim 10 \%$ of the cases of renal artery FMD), medial (80-90\%) and adventitia $(<5 \%)$. However, these categories are not mutually exclusive, since the involvement of $>1$ layer in the same diseased artery is not uncommon. ${ }^{4}$

The gold standard for diagnosis is DSA, but this invasive procedure is recommended only for patients who require revascularization during the same procedure. ${ }^{5}$ Currently, cervicocephalic FMD is classified angiographically into four subtypes: multifocal, unifocal, tubular, and atypical. ${ }^{4}$ Multifocal, with a typical string-of-beads pattern, is the most common angiographic presentation and, histologically, corresponds to medial FMD. ${ }^{5}$

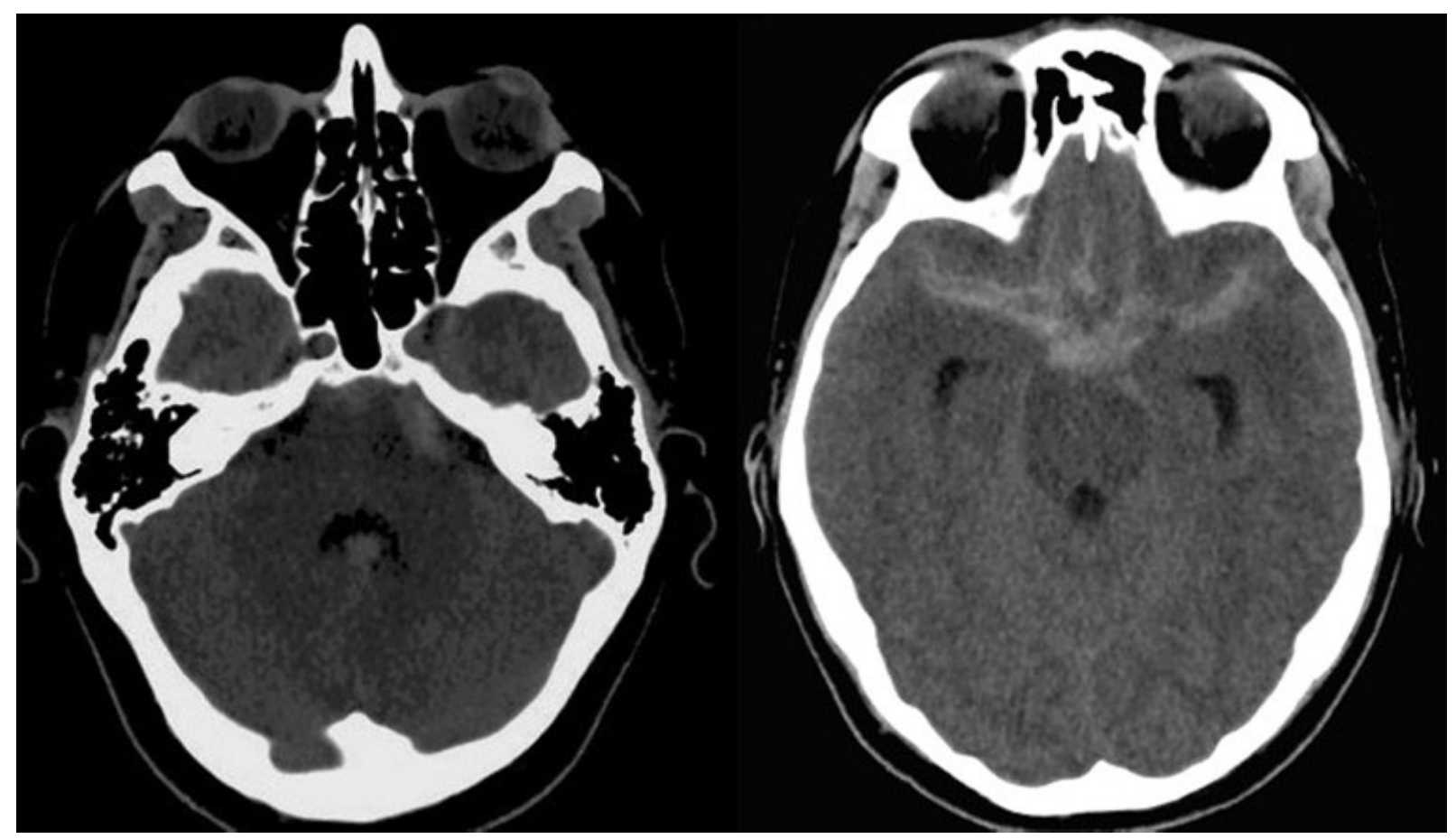

Fig. 1 Computed tomography scan shows blood in the $4^{\text {th }}$ ventricle and subarachnoid hemorrhage in the posterior fossa $(A)$ and in the base cisterns (B). 


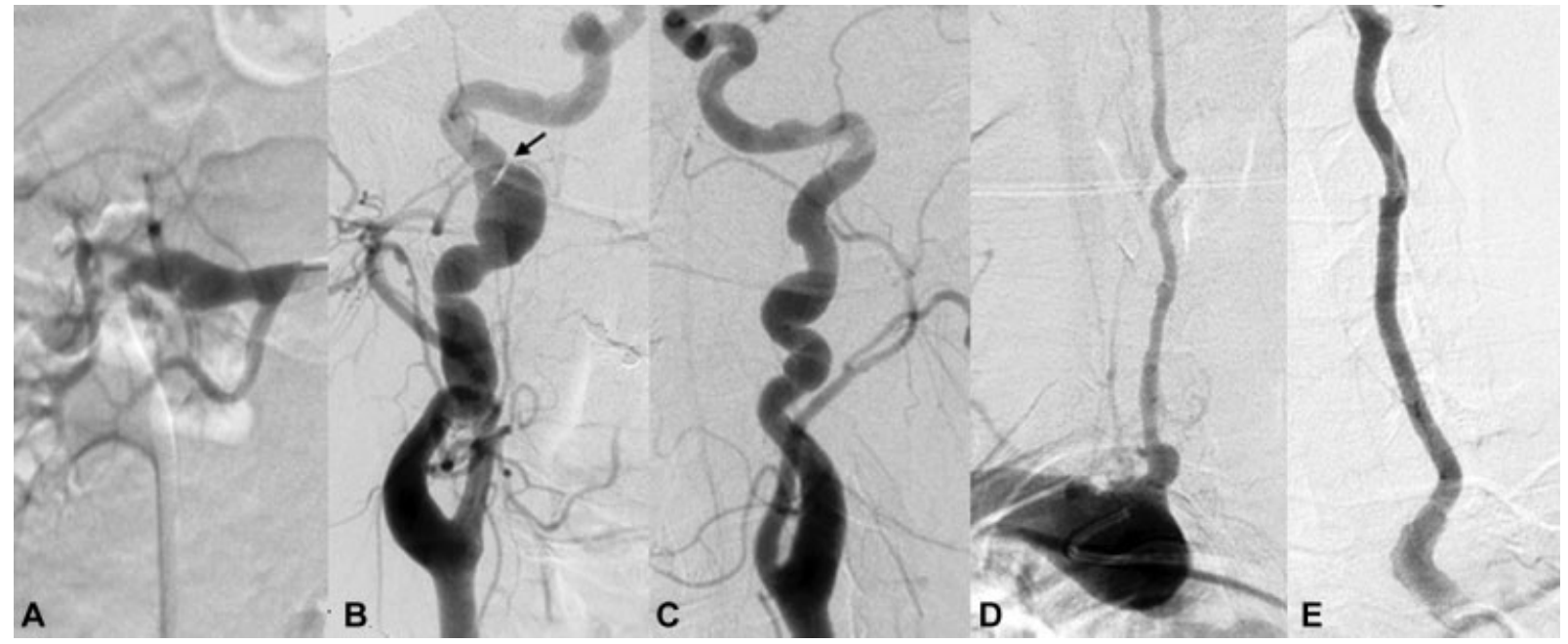

Fig. 2 Right renal artery angiogram is with ectasia (A). Cervical angiogram shows dysplastic alterations in the internal carotid artery on the right (B) and on the left (C) as well as in the right (D) and in the left (E) vertebral artery.

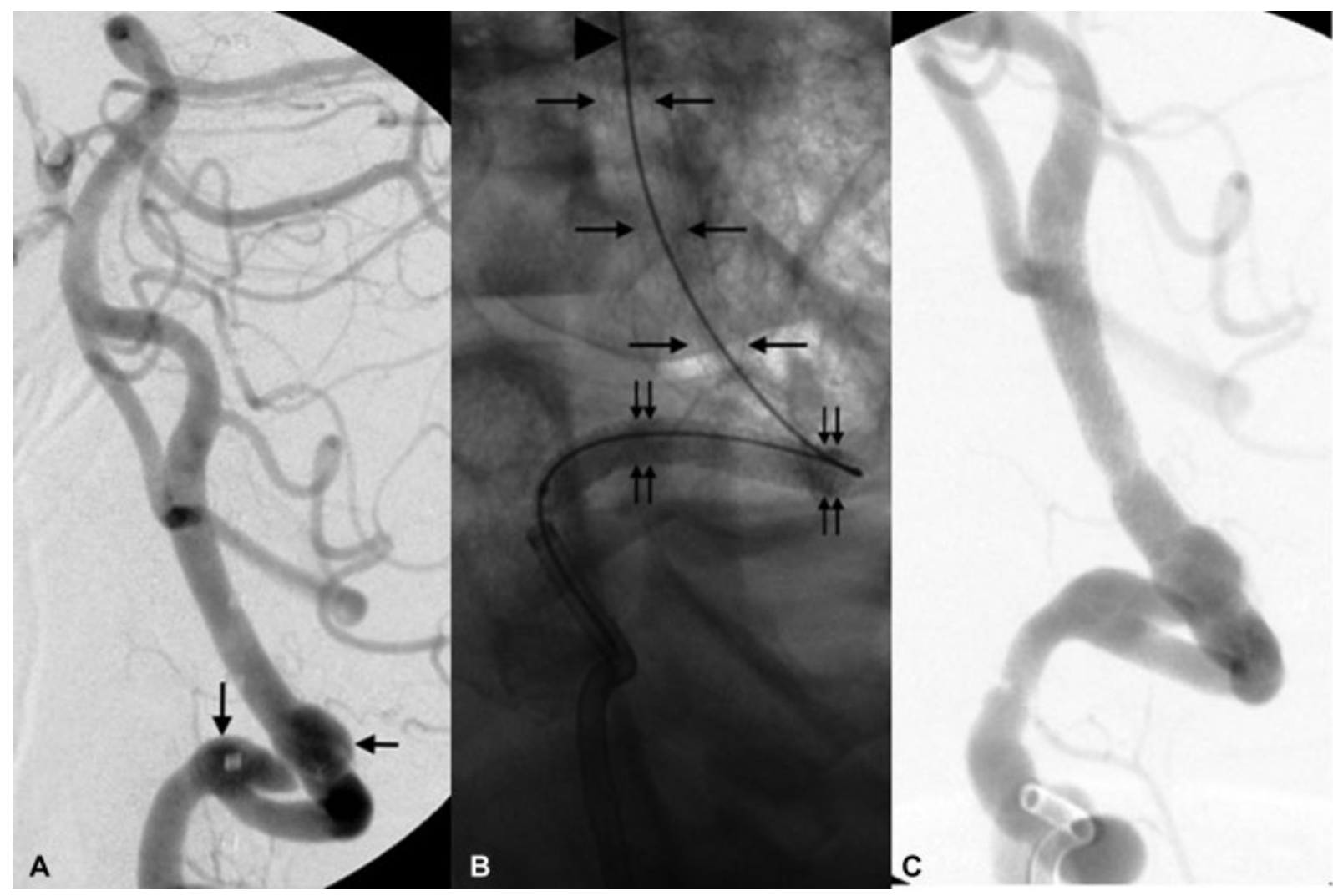

Fig. 3 Left vertebral artery angiogram shows V3-4 segments dissecting aneurysm (arrows) (A); endovascular treatment performed over a microguidewire crossing the dissection (arrowhead); with one angioplasty and stent in V4 (arrows) and another stent in V3 (double arrows) (B), covering all lesions (C).

The clinical presentation varies, depending on the location of the arterial lesions. ${ }^{3}$ The most common manifestation is renovascular hypertension secondary to involvement of the renal artery. The FMD of the cervical artery, which mainly affects the ICA, is rarely symptomatic. ${ }^{6}$ In addition, FMD is associated with a substantial risk of formation and rupture of aneurysms, as well as of arterial dissection and occlusion, ${ }^{3}$ causing a great diversity of signs and symptoms.
Fibromuscular dysplasia is more common in females: Plouin and Kadian-Dodov, in 2016, reported a prevalence of $84 \%^{5}$ and of $93.5 \%$, respectively; and Lather, in 2017 , of $94.1 \%{ }^{3}$

Fibromuscular dysplasia can affect several arterial sites, the most common being the cervical and renal. There are several reports of involvement of the mesenteric, axillary, iliac, hepatic, intracranial and, in some cases, coronary arteries. In a case series review conducted by Persu, the 
prevalence of involvement of $\geq 2$ arterial sites varied from 16 to $28 \%$. For this reason, the investigation of secondary lesions in patients with FMD is recommended, mainly in renal and cervical arteries. ${ }^{4}$

The clinical presentation of FMD is variable, not only by the multiple sites of involvement, but also by the large number of complications. Kadian-Dodov et al performed a study with 921 patients with FMD, in which 384 of them (41.7\%) had aneurysms (of the cases) and arterial dissections (25.7\%), in addition to $5.8 \%$ with simultaneous involvement. In this same study, the most commonly identified aneurysm sites reflected the most frequently affected arterial beds by the FMD: the renal (34\%) and extracranial carotid arteries (31\%). Intracranial circulation was the third most common site, occurring in $21.5 \%$ of all patients with aneurysms and in $4.7 \%$ of the 921 patients in the study. ${ }^{1}$

Lather, in 2017, performed an angiographic review of 669 women diagnosed with FMD in the USA, showing a $12.9 \%$ prevalence of intracranial aneurysms. There was no difference in the comparison between the different FMD sites: renal involvement with prevalence of $11.9 \%$, cervical with $13.7 \%$, and mixed with $13.2 \%{ }^{3}$ If we compare these results with the prevalence in the general population, with rates of $3.2 \%,{ }^{4}$ the strong relation of the development of intracranial aneurysms with all forms of FMD becomes clear.

Spontaneous cervical artery dissections are a common cause of stroke in young and middle-aged adults and are associated with FMD in $\sim 15 \%$ of the cases, ${ }^{7}$ and the most frequent dissections are in the extracranial carotid (63.7\%) and vertebral arteries $(20.7 \%)^{1}$

The management of FMD patients with drug therapy is well established, either with antiplatelet aggregators, anticoagulants or antihypertensives. Surgical or endovascular treatment is indicated in patients with symptomatic or critical FMD, and may be directed to complications. In renal FMD, options are available in the presence of renovascular hypertension, renal ischemia atrophy, or in the presence of lesions in other sites. In cervical FMD, the indications are more individualized, because of the low possibility of disease progression and, generally, they are directed to the complications. Among the surgical possibilities are the use of coils for aneurysms and percutaneous transluminal angioplasty and stents for dissections or critical FMDs. ${ }^{8}$

Because FMD is a disease of unknown cause, it remains a therapeutic challenge because it does not have a specific treatment. Currently, the therapy is directed to complications: aneurysms and arterial dissections. Although endovascular or surgical treatment is well-established for symptomatic complications or for those that cause a serious risk to the patient, more studies are needed regarding the treatment of asymptomatic lesions. The follow-up of these patients with DSA, Doppler or AngioCT/MRI is indissoluble, due to the great possibility of progression of the disease and its complications.

\section{Conclusions}

The vascular fragility of patients with FMD of cervical arteries predisposes to dissection, stenosis and occlusion, in addition to being related to intracranial aneurysms. All these vascular malformations may present spontaneous complications, with cerebral hemorrhage or stroke, or secondary to iatrogenesis. Endovascular procedures should be well indicated, and reserved only for the treatment of symptomatic lesions.

\section{Conflicts of Interests}

The authors have no conflicts of interests to declare.

\section{References}

1 Kadian-Dodov D, Gornik HL, Gu X, et al. Dissection and Aneurysm in Patients With Fibromuscular Dysplasia: Findings From the U.S. Registry for FMD. J Am Coll Cardiol 2016;68(02):176-185

2 Slovut DP, Olin JW. Fibromuscular dysplasia. N Engl J Med 2004; 350(18):1862-1871

3 Lather HD, Gornik HL, Olin JW, et al. Prevalence of Intracranial Aneurysm in Women With Fibromuscular Dysplasia: A Report From the US Registry for Fibromuscular Dysplasia. JAMA Neurol 2017;74(09):1081-1087

4 Persu A, Touzé E, Mousseaux E, Barral X, Joffre F, Plouin PF. Diagnosis and management of fibromuscular dysplasia: an expert consensus. Eur J Clin Invest 2012;42(03):338-347

5 Plouin PF, Baguet JP, Thony F, et al; ARCADIA Investigators. High Prevalence of Multiple Arterial Bed Lesions in Patients With Fibromuscular Dysplasia: The ARCADIA Registry (Assessment of Renal and Cervical Artery Dysplasia). Hypertension 2017;70(03): 652-658

6 Plouin PF, Perdu J, La Batide-Alanore A, Boutouyrie P, GimenezRoqueplo AP, Jeunemaitre X. Fibromuscular dysplasia. Orphanet J Rare Dis 2007;2:28

7 Hunt JC, Harrison EG Jr, Sheps SG, Bernatz PE, Davis GD, Bulbulian $\mathrm{AH}$. Hypertension caused by fibromuscular dyplasia of renal arteries. Postgrad Med 1965;38:53-63

8 Schievink WI. Spontaneous dissection of the carotid and vertebral arteries. N Engl J Med 2001;344(12):898-906 